\title{
Carcinoma hepatocelular en el anciano: características clínicas, análisis de supervivencia y factores pronósticos en una cohorte de pacientes españoles mayores de 75 años
}

\author{
M. Fernández-Ruiz, J. M. Guerra-Vales, J. Llenas-García y F. Colina-Ruizdelgado ${ }^{1}$ \\ Servicio de Medicina Interna. ${ }^{1}$ Registro Hospitalario de Tumores. Hospital Universitario 12 de Octubre. Universidad \\ Complutense. Madrid
}

\section{RESUMEN}

Objetivos: el carcinoma hepatocelular $(\mathrm{CHC})$ permanece mal caracterizado en pacientes de edad avanzada y comorbilidad, circunstancia que limita su manejo clínico. Pretendemos analizar la historia natural del $\mathrm{CHC}$ en mayores de 75 años y determinar los factores que condicionan su supervivencia.

Pacientes y métodos: análisis retrospectivo de 235 pacientes con $\mathrm{CHC}$ categorizados según su edad al diagnóstico: $\leq 75$ años (n $=186)$ y $>75$ años $(n=49)$. Tras comparar sus variables clínicas ( $\chi^{2}$ y t-Student), realizamos un análisis de regresión logística para determinar los factores asociados a la recepción de tratamiento locorregional (vs. sintomático); la supervivencia entre ambos grupos fue comparada mediante el test de log-rank, con posterior análisis multivariante (modelo de riesgos proporcionales de Cox).

Resultados: no se obtuvieron diferencias entre ambos grupos en su distribución por sexo, presencia de cirrosis, etiología, ChildPugh, estadio BCLC, ascitis, trombosis portal, o valores de bilirrubina, AST, ALT, yGT, LDH o hematocrito. Los pacientes de edad avanzada fueron más frecuentemente diagnosticados en presencia de manifestaciones clínicas, con enfermedad multifocal, no localiza$\mathrm{da}$, y niveles de $\alpha$-fetoproteína $>400 \mathrm{ng} / \mathrm{ml}$ (todas, $\mathrm{p}<0,05$ ). Este grupo recibió tratamiento exclusivamente sintomático en el $78 \%$ de los casos (vs. 33\% entre pacientes jóvenes), y sólo tres de ellos fueron sometidos a resección $(p<0,0001)$. La edad $>75$ años actuó como predictor de la no recepción de terapia locorregional $(\mathrm{p}<$ 0,0001). La supervivencia del grupo de mayor edad (9,8 \pm 1 meses) difirió significativamente respecto a la de los pacientes más jóvenes (25,6 \pm 2 meses) ( $p<0,00001)$. En el análisis multivariante, la edad avanzada se mantuvo como factor pronóstico de pobre supervivencia $(p=0,025)$; sin embargo, pierde su significación al estratificar dicho análisis por sub-grupos de tratamiento $(p=0,344)$.

Conclusiones: la menor supervivencia demostrada en pacientes ancianos con $\mathrm{CHC}$, al margen de diferencias en cuanto a extensión tumoral o insuficiencia hepatocelular, parece condicionada por la aplicación de abordajes terapéuticos subóptimos en esta población.

Palabras clave: Carcinoma hepatocelular. Pacientes ancianos. Edad. Pronóstico. Supervivencia. Tratamiento.

\begin{abstract}
Aims: hepatocellular carcinoma (HCC) remains poorly characterized in elderly patients with comorbid conditions, a fact that limits the clinical management of the disease. This study analyzes the natural history of HCC in patients older than 75, and determines factors that condition their survival.
\end{abstract}

Patients and methods: a retrospective analysis of 235 patients with HCC divided into 2 groups by age at diagnosis: $\leq 75$ ( $\mathrm{n}=186)$ and $>75(\mathrm{n}=49)$. After comparing their clinical variables ( $\chi^{2}$ and $t$ test), a logistic regression analysis was performed to determine factors associated with receiving locoregional treatment (versus symptomatic treatment). Survival in the 2 groups was compared using a log rank test with subsequent multivariate analysis (Cox proportional hazards model).

Results: there were no differences between groups for sex, presence of cirrhosis, etiology, Child-Pugh score, BCLC stage, presence of ascites or portal thrombosis, or bilirubin, AST, ALT, $\gamma \mathrm{GT}$, LDH or hematocrit values. Patients of advanced age were more frequently diagnosed in the presence of clinical manifestations, and had multifocal, non-localized disease and $\alpha$-fetoprotein levels $>400 \mathrm{ng} / \mathrm{mL}$ (all $\mathrm{p}<0.05$ ). This group received exclusively symptomatic treatment in $78 \%$ of cases (compared to $33 \%$ in younger patients), and only 3 of them underwent surgical resection $(p<0.0001)$. Age older than 75 was a predictive factor for not receiving locoregional therapy $(\mathrm{p}<0.0001)$. Survival in the elderly group (9.8 \pm 1 months) differed substantially from that of younger patients $(25.6 \pm 2$ months) ( $p<.00001)$. Advanced age continued to be a prognostic factor of poor survival in the multivariate analysis $(p=0.025)$, but lost significance when the analysis was stratified by treatment subgroups $(p=0.344)$.

Conclusions: the lower survival seen in elderly patients with $\mathrm{HCC}$, beyond differences in tumor extension or liver failure, seems conditioned by the use of suboptimal treatment in this population.

Key words: Hepatocellular carcinoma. Elderly patients. Age. Prognosis. Survival. Therapy.

Fernández-Ruiz M, Guerra-Vales JM, Llenas-García J, Colina-Ruizdelgado F. Carcinoma hepatocelular en el anciano: características clínicas, análisis de supervivencia y factores pronósticos en una cohorte de pacientes españoles mayores de 75 años. Rev Esp Enferm Dig 2008; 100: 625-631. 


\section{INTRODUCCIÓN}

El carcinoma hepatocelular (CHC) supone la quinta neoplasia más frecuente en varones y la octava en mujeres, con una incidencia anual estimada en 627.000 casos (5,7\% de todos los nuevos diagnósticos de cáncer) y una mortalidad prácticamente equivalente (598.000 muertes anuales), representando así la tercera causa de muerte por cáncer en el mundo (1). En nuestro medio el CHC constituye un problema de salud de creciente magnitud (2), y su prevalencia experimentará previsiblemente un incremento notable en individuos ancianos a lo largo de las próximas décadas, circunstancia condicionada por la expectativa vital de la población occidental (3). En España, y según datos referidos a 2005, la esperanza de vida al nacimiento supera los 80 años (76,9 años en varones y 83,5 en mujeres) (4). La edad media al diagnóstico del CHC en diversas cohortes nacionales ha evolucionado de forma progresiva desde la década de los ochenta (62,5 años) hasta nuestros días $(73,5$ años) $(5,6)$. Como resultado, no resulta excepcional en la práctica clínica establecer el diagnóstico de esta entidad en sujetos mayores de 75 años, con frecuente comorbilidad y fragilidad asociadas.

Diversas enfermedades neoplásicas parecen exhibir ciertas características clínicas diferenciadas en pacientes ancianos respecto a los más jóvenes, y la edad avanzada se ha vinculado frecuentemente a un impacto desfavorable en su historia natural; sin embargo, esta población resulta normalmente excluida de los ensayos clínicos en los que se evalúan nuevas líneas terapéuticas (7). Desafortunadamente, el estudio del CHC en el anciano, tanto en sus aspectos clínicos como evolutivos, ha recibido una atención limitada en las últimas décadas. Las series publicadas, al margen de la experiencia de grupos multicéntricos de determinados países europeos $(3,8,9)$, proceden en su inmensa mayoría de instituciones asiáticas (10-18), con resultados difícilmente extrapolables a medios occidentales. En nuestro conocimiento, ninguna de ellas tiene su origen en nuestro país. Además, la literatura difiere notablemente en el límite de edad empleado en la estratificación de cada cohorte, que varía desde los $50(8,18)$ ó $65(17)$ hasta los $70(3,9$ $11,13)$ y, más recientemente, 80 años $(12,14,16)$, circunstancia que limita su interpretación. Por todo ello, nos proponemos analizar la historia natural del $\mathrm{CHC}$ y su caracterización clínica en una cohorte de pacientes españoles mayores de 75 años pertenecientes a una única institución, estableciendo su supervivencia y los factores que la condicionan, para ponerla finalmente en relación con la de individuos de menor edad.

\section{MATERIAL Y MÉTODOS}

A partir del Registro de Tumores de nuestro centro, que recoge de forma prospectiva los casos incidentes de enfermedad neoplásica en el área sanitaria 11 de la Comunidad de Madrid desde 1999, realizamos un estudio longitudinal con recogida de datos retrospectiva de los pacientes con CHC diagnosticados, tratados y sometidos a seguimiento en el Hospital Universitario 12 de Octubre en el periodo comprendido entre enero de 1999 y diciembre de 2003. El diagnóstico de $\mathrm{CHC}$ quedó establecido en base a los criterios de la Asociación Europea para el Estudio del Hígado en su Conferencia de Consenso de Barcelona-2000: confirmación citohistológica, concordancia de dos técnicas de imagen sugerentes sobre una lesión focal $>2 \mathrm{~cm}$ en órgano cirrótico, o niveles séricos de alfa-fetoproteína $(\alpha-\mathrm{FP})$ $>400 \mathrm{ng} / \mathrm{ml}$ en asociación con una única técnica de imagen sugerente. De los 278 pacientes así identificados, 43 $(15,5 \%)$ fueron excluidos al carecer sus historias clínicas de la información necesaria, particularmente la relacionada con su situación funcional en el momento del diagnóstico. La cohorte analizada incluyó, por tanto, un total de 235 casos, estratificados en dos categorías: pacientes no ancianos ( $\leq 75$ años en el momento del diagnóstico del CHC) (grupo 1), y pacientes ancianos (> 75 años) (grupo 2).

Tras la revisión de su documentación clínica se obtuvieron las siguientes variables: sexo y edad; presencia de cirrosis, etiología y estadio evolutivo de la misma (ChildPugh); contexto diagnóstico (programa de seguimiento, hallazgo casual, o manifestaciones clínicas); ascitis al diagnóstico; determinaciones analíticas (hematocrito, albúmina, bilirrubina total, alanino-aminotransferasa [ALT], aspartato-aminotransferasa [AST], $\gamma$-glutamiltranspeptidasa $[\gamma \mathrm{GT}]$, fosfatasa alcalina, actividad de protrombina y $\alpha$ FP); número de nódulos; presencia de trombosis venosa portal; carácter localizado o no (criterios de Milan) (20); estadio tumoral según el modelo BCLC (Barcelona Clinic Liver Cancer) (21); y modalidad terapéutica empleada: trasplante hepático ortotópico (THO), resección quirúrgica, inyección intratumoral de alcohol (IIA), quimioembolización transarterial (QET) o tratamiento sintomático. Determinamos igualmente, cuando fue posible, la causa fundamental del fallecimiento. El objetivo primario del estudio consistió en la supervivencia global de la cohorte, definida por el intervalo transcurrido entre el diagnóstico del CHC y el fallecimiento del paciente o, en su defecto, la finalización de su periodo de seguimiento (31 de diciembre de 2004). Los pacientes perdidos durante este periodo fueron censurados a partir del último contacto conocido en nuestro centro.

\section{Análisis estadístico}

Empleamos la aplicación estadística SPSS versión 12.0 (SPSS Inc., Chicago, IL). Caracterizamos las variables cualitativas mediante la distribución de sus frecuencias absolutas y relativas. Las variables cuantitativas se ofrecen a través de su media \pm desviación estándar (DE), o bien asociadas al intervalo de confianza del $95 \%\left(\mathrm{IC}_{95}\right)$; debido a su amplia dispersión, los niveles séricos de $\alpha$ FP fueron dicotomizados ( $\leq 400 \mathrm{o}>400 \mathrm{ng} / \mathrm{ml})$. Las características entre ambos grupos fueron comparadas me- 
diante los test de $\chi^{2}$ y t de Student. A fin de determinar los factores asociados a la decisión de aplicar a cada paciente algún tipo de terapia locorregional (resección, THO, IIA o QET), frente a un tratamiento exclusivamente sintomático, realizamos un análisis multivariante mediante regresión logística con inclusión de la edad al diagnóstico, entre otras, como variable independiente. Estimamos las distribuciones de supervivencia según el modelo de Kaplan-Meier, comparando las diferencias entre grupos mediante la prueba de log-rank (Mantel-Cox) (análisis univariante). El análisis multivariante se basó en el modelo de riesgos proporcionales de Cox, aplicado sobre aquellas variables que demostraron significación estadística en el univariante. Fijamos un valor de $\mathrm{p}<0,05$ como nivel de significación estadística.

\section{RESULTADOS}

\section{Características clínicas}

Del total de 235 pacientes incluidos en el estudio, $186(79,1 \%)$ fueron clasificados como no ancianos (grupo 1) y $49(20,9 \%)$, como ancianos (grupo 2). La edad media al diagnóstico fue de 65,5 \pm 11,4 años (rango: 32-90) para la cohorte global, 61,9 $\pm 9,9$ años (rango: 32-75) en el grupo de no ancianos, y 79,1 $\pm 3,9$ años (rango: 76-90) en el de ancianos $(\mathrm{p}<0,0001)$. La proporción de pacientes mayores de 75 años respecto al total de nuevos diagnósticos anuales de $\mathrm{CHC}$ experimentó un incremento progresivo a lo largo del periodo estudiado: 9/58 (16\%) en 1999, 9/52 (17\%) en $2000,11 / 46(24 \%)$ en $2001,6 / 39(15 \%)$ en 2002 y $14 / 40(35 \%)$ en 2003 (diferencias no significativas). Las características basales de ambos grupos se exponen en la tabla I. La distribución por sexo (relación hombre:mujer) mostró, dentro del predominio masculino en ambos grupos, mayor número de mujeres ancianas $(2,06: 1)$ respecto a las más jóvenes $(2,8: 1)$, sin alcanzar esta diferencia la significación estadística. Tampoco difirieron ambos grupos en cuanto a la presencia de cirrosis, Child-Pugh, infección por virus de la hepatitis B (VHB) o C (VHC), estadificación BCLC, demostración de ascitis o trombosis venosa portal, o niveles séricos de bilirrubina, AST, ALT, $\gamma \mathrm{GT}, \mathrm{LDH}$, fosfatasa alcalina, LDH o hematocrito. El grupo de edad avanzada fue más frecuentemente diagnosticado en presencia de manifestaciones clínicas sugerentes de $\mathrm{CHC}$ y fuera, por tanto, de programas de seguimiento $(\mathrm{p}=0,001)$. Así mismo, exhibía con más probabilidad enfermedad multifocal o difusa $(\mathrm{p}=$ $0,026)$, de carácter no localizado (excediendo criterios de Milán) $(\mathrm{p}=0,006)$, y asociada a determinaciones de $\alpha$-FP $>400 \mathrm{ng} / \mathrm{ml}(\mathrm{p}=0,049)$. Los niveles séricos de albúmina fueron significativamente menores en ancia$\operatorname{nos}(\mathrm{p}=0,006)$.
Tabla I. Características basales de la cohorte según edad de diagnóstico del $\mathrm{CHC}$ ( $\leq 75$ vs. $>75$ años)

\begin{tabular}{|c|c|c|c|}
\hline & $\begin{array}{l}\text { No ancianos } \\
\quad(n=186)\end{array}$ & $\begin{array}{l}\text { Ancianos } \\
(n=49)\end{array}$ & $p$ \\
\hline Edad (años) & $61,9 \pm 9,9$ & $79,1 \pm 3,9$ & $0,000^{\circ}$ \\
\hline Sexo (hombre / mujer) & $137(73,7) / 49(26,3)$ & $33(67,3) / 16(32,7)$ & $N S^{b}$ \\
\hline Cirrosis & $162(87,1)$ & $42(85,7)$ & $N S^{b}$ \\
\hline Estadío Child-Pugh & & & $N S^{b}$ \\
\hline Child-Pugh A & $80(43)$ & $19(38,8)$ & \\
\hline Child-Pugh B & $73(39,2)$ & $23(46,9)$ & \\
\hline Child-Pugh C & $33(17,7)$ & $7(14,3)$ & \\
\hline $\mathrm{VHC}$ & $98(52,7)$ & $31(63,3)$ & $N S^{b}$ \\
\hline VHB & $23(12,4)$ & $3(6,1)$ & $N S^{b}$ \\
\hline Estadio BCLC & & & $N S^{b}$ \\
\hline BCLC A & $93(50)$ & $15(30,6)$ & \\
\hline $\mathrm{BCLC} B$ & $20(10,8)$ & $7(14,3)$ & \\
\hline $\mathrm{BCLC} C$ & $28(15,1)$ & $10(20,4)$ & \\
\hline$B C L C D$ & $45(24,2)$ & $17(34,7)$ & \\
\hline Forma de diagnóstico & & & $0,001^{\mathrm{b}}$ \\
\hline Seguimiento & $80(43)$ & $15(30,6)$ & \\
\hline Casual & $35(18,8)$ & $2(4,1)$ & \\
\hline Manifestaciones clínicas & $71(38,2)$ & $32(65,3)$ & \\
\hline Ascitis & $71(38,2)$ & $23(46,9)$ & $N S^{b}$ \\
\hline Número de nódulos & & & $0,026^{\natural}$ \\
\hline Solitario & $103(55,4)$ & $23(46,9)$ & \\
\hline Paucifocal ( $\leq 3$ nódulos) & $41(22)$ & $6(12,2)$ & \\
\hline Multifocal (> 3 nódulos) & $42(22,6)$ & $20(40,8)$ & \\
\hline Localizado (sí/no) & $78(42) / 108(58)$ & $10(20,4) / 39(79,6)$ & $0,006^{6}$ \\
\hline Trombosis venosa portal & $47(25,3)$ & $16(32,7)$ & $N S^{b}$ \\
\hline$\alpha-\mathrm{FP}(\mathrm{ng} / \mathrm{ml})^{\mathrm{c}}$ & & & $0,049^{\mathrm{b}}$ \\
\hline$\leq 400$ & $141(76,6)$ & $28(62,2)$ & \\
\hline$>400$ & $43(23,4)$ & $17(37,8)$ & \\
\hline Albúmina (g/dl) & $3,2 \pm 0,7$ & $2,9 \pm 0,7$ & $0,006^{2}$ \\
\hline Bilirrubina (mg/dl) & $3,3 \pm 4,4$ & $3,02 \pm 4,1$ & $\mathrm{NS}^{\mathrm{a}}$ \\
\hline $\operatorname{ALT}(U / /)$ & $83,4 \pm 80,7$ & $68,4 \pm 59,6$ & $N S^{a}$ \\
\hline AST (UI/I) & $104,2 \pm 94,3$ & $85,2 \pm 58,6$ & $N S^{a}$ \\
\hline$\gamma \mathrm{GT}(\mathrm{UI} / \mathrm{l})$ & $165,8 \pm 180,9$ & $201,8 \pm 282,2$ & $N S^{a}$ \\
\hline Fosfatasa alcalina (UI/I) & $333,7 \pm 272,3$ & $424,1 \pm 436,2$ & $N S^{a}$ \\
\hline $\mathrm{LDH}(\mathrm{U} / /)$ & $238,3 \pm 142,8$ & $234,7 \pm 100,4$ & $N S^{a}$ \\
\hline Actividad de protrombina (\%) & $70,2 \pm 18,9$ & $78,6 \pm 16,9$ & $0,005^{\circ}$ \\
\hline Hematocrito (\%) & $37,9 \pm 6,5$ & $38,1 \pm 5,1$ & $\mathrm{NS}^{\mathrm{a}}$ \\
\hline
\end{tabular}

BCLC: Barcelona Clinic Liver Cancer; DE: desviación estándar; NS: no significativo; VHB: virus de la hepatitis B; VHC: virus de la hepatitis C; ${ }^{\top} T e s t$ de $t$ de Student para muestras no pareadas; ${ }^{\circ}$ Test de $\chi^{2 .}$; Calculado sobre un total de 229 pacientes

\section{Estrategia terapéutica}

El abordaje terapéutico inicial difirió significativamente entre ambos grupos, como refleja la tabla II ( $\mathrm{p}<$ $0,0001)$. Sólo tres pacientes mayores de 75 años se sometieron a resección quirúrgica $(6,1 \%)$, y ninguno en este grupo fue considerado candidato a THO o IIA. En contraste, 63 de los pacientes más jóvenes (34\%) se beneficiaron de algún tipo de tratamiento potencialmente curativo (THO en 37 casos, resección en 23, e IIA en tres). La aplicación de QET fue minoritaria en el grupo de ancianos $(16,3 \%)$ en comparación con el de no ancianos (34\%). Así, la mayoría de los pacientes de edad avanzada recibió tratamiento exclusivamente sintomático para su enfermedad $(77,6 \%)$. La tabla III recoge las variables que 
Tabla II. Opción terapéutica inicial y causas de fallecimiento en ambos grupos

\begin{tabular}{lccc}
\hline & $\begin{array}{c}\text { No ancianos } \\
(n=186)\end{array}$ & $\begin{array}{c}\text { Ancianos } \\
(n=49)\end{array}$ & $p$ \\
\hline Tratamiento & $23(12,4)$ & $3(6,1)$ & $0,000^{a}$ \\
Resección quirúrgica & $37(20)$ & - & \\
THO & $3(1,6)$ & - & \\
II & $63(34)$ & $8(16,3)$ & \\
QET & $60(32,3)$ & $38(77,6)$ & \\
Sintomático & & & NS $^{\text {a }}$ \\
Causa de muerte & $30(23,1)$ & $4(8,9)$ & \\
Insuficiencia hepatocelular & $24(18,5)$ & $9(20)$ & \\
Progresión tumoral & $11(8,5)$ & $4(8,9)$ & \\
Hemorragia digestiva & $2(1,5)$ & $2(4,4)$ & \\
Infección & $4(3,1)$ & $2(4,4)$ & \\
Hemoperitoneo & $12(9,2)$ & - & \\
Otras & & & \\
Desconocida & $47(36,2)$ & $24(53,3)$ & \\
\hline
\end{tabular}

IIA: inyección intratumoral de alcohol; NS: no significativo; QET: quimioembolización transarterial; THO: trasplante hepático ortotópico; 'Test de $\chi^{2}$; . Porcentajes calculados sobre 130 fallecimientos en el grupo 1 y 45 en el grupo 2; Incluye: neoplasia primaria mútiple (2), complicación post-QET (2), disfunción primaria del injerto, peritonitis bacteriana espontánea, pancreatitis aguda enólica, ictus y fractura de cadera.

Tabla III. Análisis de regresión logística de variables predictoras de la recepción de tratamiento locorregional (vs. tratamiento exclusivamente sintomático)

\begin{tabular}{|c|c|c|c|}
\hline & $\begin{array}{l}\text { OR de recibir } \\
\text { tratamiento }\end{array}$ & $\begin{array}{c}\text { Intervalo de } \\
\text { confianza (95\%) }\end{array}$ & $p$ \\
\hline $\begin{array}{l}\text { Año de diagnóstico } \\
2001-2003 \text { vs. } 1999-2000\end{array}$ & 1,43 & $0,71-2,86$ & NS \\
\hline $\begin{array}{l}\text { Sexo } \\
\text { Mujer vs. varón }\end{array}$ & 0,72 & $0,33-1,57$ & NS \\
\hline Edad & & & \\
\hline $\begin{array}{l}\text { Anciano vs. no anciano } \\
\text { Niveles de } \alpha F P\end{array}$ & 0,12 & $0,05-0,30$ & 0,000 \\
\hline $\begin{array}{l}>400 \text { vs. } \leq 400 \mathrm{ng} / \mathrm{ml} \\
\text { Cirrosis }\end{array}$ & 0,99 & $0,45-2,19$ & NS \\
\hline $\begin{array}{l}\text { Presente vs. ausente } \\
\text { Localizado }\end{array}$ & 0,24 & $0,07-0,77$ & 0,017 \\
\hline $\begin{array}{l}\text { No vs. sí } \\
\text { Estadio } B C L C\end{array}$ & 0,28 & $0,10-0,74$ & 0,01 \\
\hline B vs. A & 0,56 & $0,16-1,91$ & NS \\
\hline C VS. A & 0,19 & $0,06-0,64$ & 0,007 \\
\hline D vs. A & 0,13 & $0,05-0,35$ & 0,000 \\
\hline
\end{tabular}

BCLC: Barcelona Clinic Liver Cancer; NS: no significativo; OR: odds ratio.

demostraron predecir, en el análisis multivariante mediante regresión logística, la elección de algún tipo de tratamiento locorregional para el CHC (THO, resección, IIA o QET) en oposición al manejo exclusivamente sintomático de la entidad. La edad $>75$ años se asoció de forma manifiesta ( $<<0,0001)$ a esta última opción (OR para la recepción de tratamiento activo $=0,12$; IC95: 0,050,30 ); la presencia de cirrosis, el carácter no localizado de la enfermedad, y una estadificación avanzada en el modelo BCLC mantuvieron igualmente significación estadística, en contraste con otras variables incluidas en el análisis (sexo, periodo de diagnóstico o nivel de $\alpha$-FP).

\section{Supervivencia y causas de muerte}

Finalizado el periodo de estudio, habían fallecido 175 pacientes (74,5\% de la cohorte global); 45 en el grupo de ancianos $(91,8 \%)$ y 130 en el de no ancianos $(69,9 \%)$ (p $=0,002)$. Once pacientes $(4,7 \%)$ fueron perdidos durante el seguimiento. La probabilidad de supervivencia estimada a los 12, 24 y 36 meses del diagnóstico fue de 52,6; 39,7 y $30,2 \%$ en el grupo de menor edad, y 38,8; 7 y $2,3 \%$ en el de mayor edad, respectivamente. La figura 1 muestra las curvas Kaplan-Meier de ambos grupos; la supervivencia en pacientes ancianos $(9,8 \pm 1,4$ meses) difirió significativamente de la de los más jóvenes $(25,6 \pm$ $2,0$ meses) ( $p<0,00001)$. Las causas de fallecimiento se detallan en la tabla II; el fracaso hepatocelular $(23,1 \%)$ y la progresión tumoral (20\%) fueron las más frecuentes en pacientes de menor y mayor edad, respectivamente. El análisis univariante seleccionó como significativos los siguientes factores pronósticos: contexto diagnóstico ( $\mathrm{p}=$ 0,003), edad, Child-Pugh, BCLC, presencia de ascitis, invasión venosa portal, número de nódulos tumorales, nivel sérico de $\alpha$-FP y carácter localizado o no (según criterios de Milán) (todos, $\mathrm{p}<0,00001)$. La tabla IV, por su parte, ofrece el resultado del análisis multivariante: la edad $>75$ años, un grado Child-Pugh B o C, el carácter no localizado de la enfermedad, el nivel de $\alpha$-FP $>400 \mathrm{ng} / \mathrm{ml}$, y una estadificación en el modelo BCLC avanzada (C) o terminal (D), mantuvieron significación estadística en el modelo de Cox. Sin embargo, cuando dicho análisis es estratificado en función del tipo de tratamiento aplicado (resección quirúrgica, QET o sintomático), la edad al diagnóstico queda excluida del modelo $(\mathrm{p}=0,34)$, al tiempo que se conservan el resto de las variables anteriormente seleccionadas. Así, no fue posible demostrar diferencias estadísticamente significativas en la superviven-

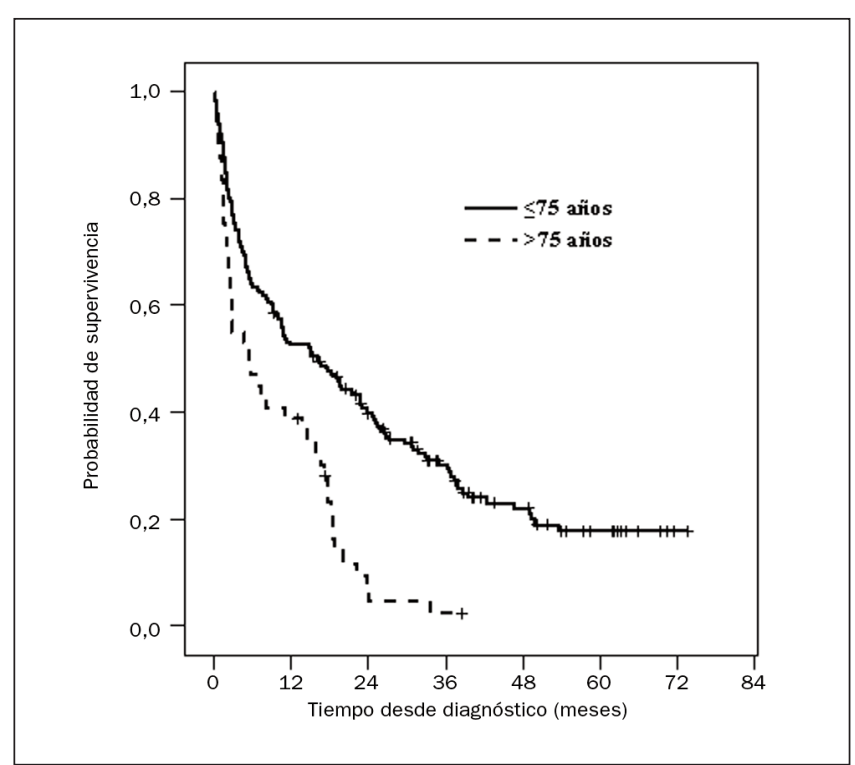

Fig. 1. Curvas de supervivencia acumulada (Kaplan-Meier) de los dos grupos de pacientes $(p<0,00001)$. 
Tabla IV. Análisis multivariante de supervivencia en la cohorte global $(n=235)$ (modelo de Cox)

\begin{tabular}{|c|c|c|c|}
\hline Variable & Riesgo relativo & $\begin{array}{c}\text { Intervalo de } \\
\text { confianza (95\%) }\end{array}$ & $p$ \\
\hline \multicolumn{4}{|l|}{ Edad } \\
\hline $\begin{array}{l}\text { Anciano vs. no anciano } \\
\text { Child-Pugh }\end{array}$ & 1,53 & $1,05-2,21$ & 0,025 \\
\hline $\begin{array}{l}\text { B-C vs. A } \\
\text { Estadio BCLC }\end{array}$ & 1,72 & $1,09-2,71$ & 0,019 \\
\hline B vs. A & 1,07 & $0,58-1,97$ & NS \\
\hline C vs. A & 2,81 & $1,54-5,13$ & 0,001 \\
\hline D vs. A & 6,75 & $3,87-11,75$ & 0,000 \\
\hline \multicolumn{4}{|l|}{ Contexto diagnóstico } \\
\hline $\begin{array}{l}\text { Seguimiento vs. clínica o casual } \\
\text { Ascitis }\end{array}$ & 0,80 & $0,58-1,10$ & NS \\
\hline $\begin{array}{l}\text { Presente vs. ausente } \\
\text { Niveles de } \alpha-F P\end{array}$ & 0,79 & $0,51-1,24$ & NS \\
\hline $\begin{array}{l}>400 \text { vs. } \leq 400 \mathrm{ng} / \mathrm{ml} \\
\text { Invasión portal }\end{array}$ & 1,67 & $1,18-2,37$ & 0,004 \\
\hline $\begin{array}{l}\text { Presente vs. ausente } \\
\text { Número de nódulos }\end{array}$ & 0,77 & $0,50-1,17$ & NS \\
\hline $\begin{array}{l}\text { Multifocal vs. solitario } \\
\text { Localizado (criterios de Milán) }\end{array}$ & 1,16 & $0,80-1,67$ & NS \\
\hline No vs. sí & 2,92 & $1,91-4,47$ & 0,000 \\
\hline
\end{tabular}

cia correspondiente a ambos grupos de edad cuando fueron comparadas de forma estratificada dentro de cada uno de los tres grupos de tratamiento antes citados (curvas no mostradas).

\section{DISCUSIÓN}

A pesar de su creciente relevancia médica y sanitaria, son limitados los estudios que aborden específicamente aspectos clínicos, terapéuticos y pronósticos propios del CHC en pacientes ancianos (3,8-18); además, de forma mayoritaria esta literatura procede de medios no occidentales y ofrece difícil generalización a nuestro entorno. No obstante, su revisión permite establecer algunas características comunes a esta población, entre las que destaca el mayor número de mujeres $(10,11,13,16,18)$ y la menor prevalencia de infección por VHB $(3,8,10,12,15-18)$, en comparación con individuos de inferior edad. Estos resultados quedan reproducidos en nuestra serie, si bien con diferencias que no alcanzan la significación estadística. La caracterización de los niveles de $\alpha$-FP muestra discrepancia entre cohortes asiáticas, que presentan cifras menos elevadas del marcador en ancianos $(10,13,17)$, y europeas, con resultados opuestos $(3,8)$; en nuestra experiencia, la determinación de $\alpha$-FP superior a $400 \mathrm{ng} / \mathrm{ml}$ fue más frecuente en pacientes de edad avanzada y actuó como predictor independiente de mal pronóstico, comportamiento previamente descrito en la literatura (22). Algunos autores han demostrado, partiendo del análisis de piezas de hepatectomía, la presencia de factores morfológicamente favorables en el CHC de sujetos ancianos, que exhibirían menor tamaño tumoral, mejor diferenciación histológica, frecuente encapsulación y una estadificación TNM (tumour-node-metastasis) menos avanzada $(10,15,17)$. Significativamente, la comparación entre ambas franjas etarias de variables relacionadas tanto con la función hepatocelular (Child-Pugh, presencia de cirrosis o ascitis) como con el grado de extensión tumoral (trombosis venosa portal, metástasis extrahepáticas, estadio CLIP [Cancer of the Liver Italian Program]) no arroja diferencias significativas en la mayor parte de las series publicadas, incluida la presente $(3,8,10,11,13,14,16)$. En nuestra cohorte tampoco demostramos que los pacientes de mayor edad asocien una estadificación adversa en el modelo BCLC, si bien este grupo presentó con más frecuencia enfermedad multifocal y no localizada al diagnóstico, al exceder los criterios de Milán. Este hallazgo puede ser vinculado a la menor inclusión de sujetos ancianos portadores de cirrosis en programas de seguimiento y diagnóstico precoz de $\mathrm{CHC}$, a la vista de las diferencias obtenidas en nuestra serie respecto al contexto diagnóstico (por seguimiento, casual o basado en síntomas) entre ambos grupos. A este respecto, Trevisani y cols. demostraron en un amplio estudio retrospectivo en cirróticos mayores de 70 años una mayor supervivencia global al cabo de tres años en el grupo sometido a seguimiento periódico mediante ultrasonografía y determinación de $\alpha$-FP, respecto al que sólo recibió investigación clínica "a demanda" (9).

El estudio del impacto que la edad de diagnóstico ejerce sobre la historia natural del CHC muestra, tanto en el análisis univariante como en el multivariante no ajustado por tratamiento, una supervivencia global significativamente menor en los pacientes ancianos de nuestra cohorte; en esta línea, Pignata y cols. obtienen resultados similares en su grupo italiano, comunicando una supervivencia media de 18,8 frente a 14,3 meses en sujetos menores y mayores de 70 años, respectivamente (3). En contraste, otros autores asiáticos no demuestran diferencias relevantes en el pronóstico de sus respectivas series, también sometidas a un manejo terapéutico multimodal $(14,16)$; cabe señalar, no obstante, que la proporción de pacientes ancianos que recibió tratamiento activo (QET, IIA u otras técnicas de ablación local) en ambas cohortes no occidentales fue similar a la del grupo de menor edad y notablemente superior, por tanto, a la recogida en instituciones europeas.

La edad avanzada y su carga de fragilidad y comorbilidad motivan frecuentemente la adopción de actitudes nihilistas basadas en aproximaciones terapéuticas subóptimas en sujetos con enfermedad neoplásica (7); diversos autores han destacado la relevancia del infratratamiento a la hora de justificar el pronóstico desfavorable de los pacientes ancianos afectos de cáncer de pulmón (24), mama (25) u ovario (26), entre otros. El análisis mediante regresión logística en nuestra serie demuestra que la edad al diagnóstico superior a 75 años limitó de forma independiente la probabilidad de recibir algún tipo de opción terapéutica potencialmente curativa. Por otra parte, tras estratificar por grupos de tratamiento el análisis multivariante de 
supervivencia, esta variable perdió su significación estadística en el modelo de Cox. Dichos hallazgos reproducen los recientemente comunicados por el grupo CLIP en una cohorte multicéntrica de 650 pacientes, a partir de los cuales concluyen sus autores en señalar el infratratamiento como principal condicionante pronóstico del $\mathrm{CHC}$ en el anciano (3). Desde un punto de vista experimental la edad avanzada se acompaña de un deterioro progresivo de la función hepática mediado por diversos mecanismos celulares (lesión mitocondrial, defectos en la cadena respiratoria o insuficiencia de las células de Kupffer) $(11,23)$. En esta circunstancia, asociada a su limitada expectativa vital y a la concurrencia de enfermedades comórbidas, reside la frecuente exclusión del anciano de abordajes considerados agresivos, ya sean técnicas quirúrgicas u otros procedimientos de ablación locorregional con impacto demostrado sobre la historia del CHC, como la IIA (27) o la QET (28). Sin embargo, son varios los estudios que demuestran el beneficio en términos de supervivencia que la resección hepática ofrece a grupos seleccionados de pacientes de edad avanzada, con un riesgo quirúrgico aceptable y una mortalidad hospitalaria (entre el 0 y el 10\%, según las series) similar a la de individuos más jóvenes $(10,11,15,17)$. Hanazaki y cols. aportan una de las cohortes más amplias de la literatura, con 103 pacientes mayores de 70 años sometidos a técnicas que van desde la segmentectomía hasta la hepatectomía derecha ampliada, y obtienen una supervivencia global a los cinco años del $42,2 \%$, que no difiere de forma significativa de la observada en el grupo de no ancianos $(40,0 \%)$; la presencia de cirrosis e invasión vascular fueron seleccionados como factores independientes de pobre pronóstico (11). Poon y cols. describen en su institución la reducción progresiva de la tasa de mortalidad post-quirúrgica correspondiente a dos cohortes históricas de mayores de 70 años sometidos a hepatectomía (25\% en el periodo 1989 1992 frente a 4\% en 1993-1997) (10). Resultados similares en términos de tolerancia y eficacia han sido demostrados en relación con opciones no quirúrgicas del CHC a edades avanzadas, ya sean técnicas percutáneas (13) o procedimientos de QET (10). Teratani y cols. obtienen una supervivencia a los 5 años del $27 \%$ en una serie de 137 sujetos mayores de 70 años sometidos a IIA, equiparable a la del grupo de control de menor edad; sorprendentemente, los autores no comunican diferencias significativas al analizar específicamente la mortalidad por causas extrahepáticas en ambos grupos de pacientes (13).

Desgraciadamente, el carácter retrospectivo de nuestro estudio dificulta la valoración de determinados aspectos, como la situación funcional (performance status) basal del paciente o su comorbilidad, de relevancia demostrada tanto en el pronóstico del paciente oncológico (29) como en la elección y tolerancia de su tratamiento (30). Por ejemplo, la variabilidad observada en nuestra cohorte respecto a los niveles séricos de albúmina $(2,9 \mathrm{~g} / \mathrm{dl}$ en ancianos frente a 3,2 g/dl en no ancianos) resultaría atribuible, más allá de diferencias en la hepatopatía crónica de base de ambos grupos, a factores propios de la edad avanzada, tales como la malnutrición u otras comorbilidades digestivas. Por otra parte, en el ya citado trabajo de Pignata y cols. la potencia de la edad avanzada como predictor de la recepción de tratamiento activo se mantuvo aun después de ajustar el análisis multivariante con la inclusión del performance status como variable independiente (3).

En conclusión, el presente estudio, con las limitaciones propias de su diseño, demuestra que los pacientes mayores de 75 años afectos de CHC en nuestro medio no difieren sustancialmente en el grado de insuficiencia hepatocelular (Child-Pugh) o estadificación tumoral (BCLC) respecto a los más jóvenes; sin embargo, se benefician en menor medida de la inclusión en programas específicos de seguimiento y exhiben una supervivencia significativamente menor. Este pronóstico desfavorable quedaría explicado, al menos parcialmente, por el abordaje terapéutico subóptimo al que se ve sometida esta población, a pesar de la evidencia que sustenta el beneficio y la tolerancia de las técnicas de resección hepática o ablación locorregional en grupos seleccionados de edad avanzada.

\section{BIBLIOGRAFÍA}

1. Parkin DM, Bray F, Ferlay J, Pisan P. Global cancer statistics, 2002. CA Cancer J Clin 2005; 55: 74-108.

2. El-Serag HB, Davila JA, Petersen NJ, McGlynn KA. The continuing increase in the incidence of hepatocellular carcinoma in the United States: an update. Ann Intern Med 2003; 139: 817-23.

3. Pignata S, Gallo C, Daniele B, Elba S, Giorgio A, Capuano G, et al. Characteristics at presentation and outcome of hepatocellular carcinoma (HCC) in the elderly. A study of the Cancer of the Liver Italian Program (CLIP). Crit Rev Oncol Hematol 2006; 59: 243-9.

4. Instituto Nacional de Estadística. Indicadores demográficos básicos. Esperanza de vida al nacimiento [consultado 30-10-2007]: Disponible en: http://www.ine.es/inebmenu/mnu_analisis.htm.

5. Calvet X, Bruix J, Brú C, Ginés P, Vilana R, Solé M, et al. Natural history of hepatocellular carcinoma in Spain. Five year's experience in 249 cases. J Hepatol 1990; 10: 311-7.

6. García-Torres ML, Zaragoza A, Giner R, Primo J, del Olmo JA. Incidencia y factores epidemiológicos del carcinoma hepatocelular en Valencia durante el año 2000. Rev Esp Enferm Dig 2003; 95: 381-4.

7. Bernardi D, Errante D, Tirelli U, Salvagno L, Bianco A, Fentiman IS. Insight into the treatment of cancer in older patients: developments in the last decade. Cancer Treat Rev 2006; 32: 277-88.

8. Trevisani F, D'Íntino PE, Grazi GL, Caraceni P, Gasbarrini A, Colantoni A, et al. Clinical and pathologic features of hepatocellular carcinoma in young and older Italian patients. Cancer 1996; 77: 2223-32.

9. Trevisani F, Cantarini MC, Labate AM, De Notariis S, Rapaccini G, Farinati F, et al. Surveillance for hepatocellular carcinoma in elderly Italian patients with cirrhosis: effects on cancer staging and patient survival. Am J Gastroenterol 2004; 99: 1470-6.

10. Poon RTP, Fan ST, Lo CM, Liu CL, Ngan H, Ng IO, et al. Hepatocellular carcinoma in the elderly: results of surgical and nonsurgical management. Am J Gastroenterol 1999; 94: 2460-6.

11. Hanakazi K, Kajikawa S, Shimozawa N, Shimada K, Hiraguri M, Koide N, et al. Hepatic resection for hepatocellular carcinoma in the elderly. J Am Coll Surg 2001; 192: 38-46.

12. Hazama H, Omagari K, Matsuo I, Masuda J, Ohba K, Sakimura K, et al. Clinical features and treatment of hepatocellular carcinoma in eight patients older than eighty years of age. Hepatogastroenterology 2001; 48: 1692-6. 
13. Teratani T, Ishikawa T, Shiratori Y, Shiina S, Yoshida H, Imamura M, et al. Hepatocellular carcinoma in elderly patients: beneficial therapeutic efficacy using percutaneous ethanol injection therapy. Cancer 2002; 95: 816-23.

14. Dohmen K, Shirahama M, Shigematsu H, Irie K, Ishibashi H. Optimal treatment strategy for elderly patients with hepatocellular carcinoma. J Gastroenterol Hepatol 2004; 19: 859-65.

15. Yeh CN, Lee WC, Jeng LB, Chen MF. Hepatic resection for hepatocellular carcinoma in elderly patients. Hepatogastroenterology 2004; 51: $219-23$.

16. Tsukioka G, Kakizaki S, Sohara N, Sato K, Takagi H, Arai H, et al. Hepatocellular carcinoma in extremely elderly patients: an analysis of clinical characteristics, prognosis and patient survival. World J Gastroenterol 2006; 12: 48-53.

17. Zhou L, Rui JA, Wang SB, Chen SG, Qu Q, Chi TY, et al. Clinicopathological features, post-surgical survival and prognostic indicators of elderly patients with hepatocellular carcinoma. Eur J Surg Oncol 2006; 32: 767-72.

18. Fujishima T, Ishikawa T, Shiratori Y, Kanda M, Tateishi R, Akamatsu M, et al. Age-related comparison of the profiles of patients with hepatocellular carcinoma. Hepatogastroenterology 2006; 53: 913-8.

19. Bruix J, Sherman M, Llovet JM, Beaugrand M, Lencioni R, Burroughs $\mathrm{AK}$, et al. Clinical management of hepatocellular carcinoma. Conclusions of the Barcelona-2000 EASL conference. European Association for the Study of the Liver. J Hepatol 2001; 35: 421-430.

20. Mazzaferro V, Regalia E, Doci R, Andreola S, Pulvirenti A, Bozzetti F, et al. Liver transplantation for the treatment of small hepatocellular carcinomas in patients with cirrhosis. N Engl J Med 1996; 334: 693-9.

21. Llovet JM, Brú C, Bruix J. Prognosis of hepatocellular carcinoma: the BCLC staging classification. Semin Liver Dis 1999; 19: 329-38.

22. Peng SY, Chen WJ, Lai PL, Jeng YM, Sheu JC, Hsu HC. High $\alpha$-feto- protein level correlates with high stage, early recurrence and poor prognosis of hepatocellular carcinoma: significance of hepatitis virus infection, age, p53 and $\beta$-catenin mutations. Int J Cancer 2004; 112: 44-50.

23. Hoshida Y, Ikeda K, Kobayashi M, Suzuki Y, Tsubota A, Saitoh S, et al. Chronic liver disease in the extremely elderly of 80 years or more: clinical characteristics, prognosis and patient survival analysis. $\mathrm{J} \mathrm{He}$ patol 1999; 31: 860-6.

24. $\mathrm{Ng} \mathrm{R}$, de Boer R, Green MD. Undertreatment of elderly patients with non-small-cell lung cancer. Clin Lung Cancer 2005; 7: 168-74.

25. Bouchardy C, Rapiti E, Fioretta G, Laissue P, Neyroud-Caspar I, Schafer P, et al. Undertreatment strongly decreases prognosis of breast cancer in elderly women. J Clin Oncol 2003; 21: 3580-7.

26. Petignat P, Fioretta G, Verkooijen HM, Vlastos AT, Rapiti E, Bouchardy $\mathrm{C}$, et al. Poorer survival of elderly patients with ovarian cancer: a population-based study. Surg Oncol 2004; 13: 181-6.

27. Yamamoto J, Okada S, Shimada K, Okusaka T, Yamasaki S, Ueno H, et al. Treatment strategy for small hepatocellular carcinoma: comparison of long-term results after percutaneous ethanol injection therapy and surgical resection. Hepatology 2001; 34: 707-13.

28. Llovet JM, Bruix J. Systematic review of randomized trials for unresectable hepatocellular carcinoma: chemoembolization improves survival. Hepatology 2003; 37: 429-42.

29. Wedding U, Röhrig B, Klippstein A, Pientka L, Höffken K. Age, severe comorbidity and functional impairment independently contribute to poor survival in cancer patients. J Cancer Res Clin Oncol 2007; 133: $945-50$.

30. Seve P, Sawyer M, Hanson J, Broussolle C, Dumontet C, Mackey JR. The influence of comorbidities, age, and performance status on the prognosis and treatment of patients with metastatic carcinomas of unknown primary site: a population-based study. Cancer 2006; 106 : 2058-66. 